\title{
Peeling back the solid layers
}

The surfaces of plants, from lotus leaves to tropical pitcher plants, have inspired the fabrication of liquid-infused materials that can repel unwanted liquids and solids. However, the durability of these surfaces is often poor because of evaporation or leakage. Overcoming these limitations, Jianyong Lv, Jianjun Wang and co-workers report a solid organogel with a regenerable sacrificial alkane surface layer in Advanced Materials.

Despite great efforts in the development of repellent surfaces, the fabrication of robust anti-icing materials remains challenging. The focus for Wang and colleagues is the development of materials with ultralow ice adhesion so that ice formed on the surface can shed owing to its own gravity or the wind. This property has been demonstrated using liquid-infused porous surfaces that work by confining liquids in a textured solid, but their durability is a key concern because of migration and evaporation of the infused liquid, which can contaminate neighbouring surfaces. "To overcome these problems, we came up with a solid surface wax layer inspired by the epicuticular wax on the surface of land plant

If foreign

materials

(for example, ice, graffiti and paints) are deposited on the surface, they can be easily removed together with the surface wax layer leaves," explains Wang. The specific feature of the epicuticular wax layer that interested the researchers is its ability to self-heal after being scratched or damaged. To mimic this behaviour, the group prepared an organogel with a sacrificial solid alkane layer that can regenerate, and that can not only be used in anti-icing, but also in antigraffiti and antifouling applications.

The organogel was prepared by swelling a crosslinked poly(dimethylsiloxane) (PDMS) network with molten alkane at high temperature. When the temperature is reduced, the solubility of the alkane molecules decreases, leading to supersaturation and deposition in the polymer network. The formation of crystalline alkane particles, or 'solid wax', causes the polymer network to deform. "Owing to the stress exerted by its surrounding, the solid wax can ooze out, and thus a thin solid wax layer forms on the surface," describes Wang. "Consequently, if foreign materials (for example, ice, graffiti and paints) are deposited on the surface, they can be easily removed together with the surface wax layer." After removal, the surface layer can regenerate after only 24 hours owing to the alkane concentration gradient formed from the bulk to the surface. As alkane molecules continue to diffuse out, alkane crystals start to form at the PDMS/surface layer interface; this could lead to a weaker interaction between PDMS and the alkane surface layer, explaining why it is easy to remove.

The ease of removing foreign materials was confirmed by spraying aerosol paint (commonly used for graffiti) on the surface of the organogel, which could then be wiped off with a cleaning cloth. Similarly, water-based paint, nitrolacquer paint and alkyd paint deposited on the organogel surface could be peeled off; the adhesion strength of each paint on the organogel surface is three orders of magnitude lower than when the paints are deposited on an aluminium plate. In addition, ice-adhesion tests demonstrated that the adhesion value remains unchanged over a wide temperature range from -20 to $-70^{\circ} \mathrm{C}$, and after 20 icing-de-icing cycles, the adhesion strength is unaffected. These results verify the durability of the solid organogel material.

Looking beyond this proofof-concept, the researchers hope to see this system adopted in a range of anti-icing, antigraffiti and antifouling settings. "We are now working together with our industry collaborators to identify commercial applications on the basis of these findings," concludes Wang. Angela Babi 